\title{
Muscle Status Biomarkers and Athletes: A Review
}

\author{
Monika
}

\begin{abstract}
Aim: The aim of this review was to discuss the role of biomarkers of muscle status of athletes. Results: The research in the field of exercise science has identified various biomarkers for assessing athlete's health, performance, and recovery during training. However, there are biomarkers which changes in individual's participating in physical activity and exercise training programs. In the present review an approach was to review the current literature of molecular science and determined a set of validated biomarkers of muscle status and recovery of athletes that could be used by coaches and trainers. Conclusion: The present review will help sport scientists, coaches, trainers, clinical sport professionals, researchers, and athletes to better understand how to monitor biomarkers of muscle status of athletes, as they design training cycles that elicit maximal improvements in performance while minimizing overtraining and injury risk.

Monika

Assistant Professor

Department of Biotechnology

Key Words: BCAA, DHEA, SHBG, Taurine, T:C ratio
\end{abstract}

Mata Gujri College, Fatehgarh Sahib , Punjab, India

E-mail: monika187@rediffmail.com

DOI: 10.18376/jesp/2018/v14/i1/111299

\section{Introduction}

The composition, metabolic capacity, structure, size, and contractile indices of skeletal muscle tissue (i.e. quality of skeletal muscle tissue) are an important aspect of athletic health and performance. Power, fatigue, strength, and endurance in athletes are directly affected by muscle condition or the fatigue and recovery state of the muscle. Furthermore, not enough recovery from exercise-induced muscle damage caused by training impairs performance, likely because of increased sense of effort, reduced exercise tolerance, reduced strength, and reduced power. Monitoring indices of muscle status will help athletes to alter their training/competition and recovery programme to optimize performance. Blood-based biomarker muscle status assessment should focus on endocrine regulation of muscle adaptations/repair, metabolic homeostasis (anabolic-catabolic balance, protein/amino acid deficiencies, and substrate availability), muscle damage, and muscle excitability. There are well-validated markers related to fatigue, recovery, protein synthesis, or fueling strategies, which are all major athlete concerns. Because hormone and amino acid concentrations in the blood are highly variable among individuals, these types of biomarkers are best assessed by analyzing progressive increases/decreases away from a baseline measure for each person. This requires monitoring for these types of biomarkers at multiple time points throughout training, off-season, and competition cycles. To observe chronic changes across a season, athletes may be tested every 4-6 weeks under similar conditions (i.e. fasted, in the morning, before training, the day after a rest day or similar training day).

\section{Hormonal Response}

An appropriate hormonal signaling is necessary for the physiological adaptations to exercise training. Dependent on the magnitude of the training stimulus, often defined by acute program variables such as load, volume, duration, modality, and rest, hormones elicit specific training 


\section{Journal of Exercise Science \& Physiotherapy, Vol. 14, No. 1 (January to June), 2018 ISSN: 0973-2020 (Print) I I OR Impact Factor = 5.23 UGC Approved [no.7485] ISSN: 2454-6089 (Online)}

adaptations. Testosterone, Cortisol, Dehydroepiandrosterone (DHEA), Growth hormone (GH), Insulin-like growth factor 1 (IGF-1), Sex-hormone binding globulin, and Luteinizing hormone (LH) are among the key hormones demonstrated to be significant to athletes.

Testosterone is required for promoting protein synthesis, red blood cell production, and glycogen replenishment and for reducing protein breakdown. Decreased testosterone levels accompanied by decreased performance, energy, or strength observed during a training season may indicate that that training volume is too high. In this case, an athlete may benefit from temporarily reducing training volume.

Cortisol works antagonistically to testosterone, inhibiting protein synthesis by interfering with testosterone's binding to its androgen receptor and by blocking anabolic signaling through testosterone-independent mechanisms. When chronically elevated, cortisol is catabolic and immunosuppressive leading to circumstances that make it more difficult for an athlete to build/maintain muscle mass and recover from training. In addition to monitoring testosterone and cortisol separately, monitoring their relative levels ( $\mathrm{T}: \mathrm{C}$ ratio) during a training season may provide a relative indication of anabolic-catabolic balance, especially in male athletes (Urhausen et al.1995). T:C ratio is considered more sensitive to training stresses than either measure alone. A prolonged decrease in $\mathrm{T}: \mathrm{C}$ is associated with detriments to performance through increased proteolysis (muscle protein breakdown) and decreased protein synthesis. A 30\% decrease in T:C has been suggested as an indicator of insufficient recovery (Banfi et al.1993), whereas a value of $0.35 \times 10^{-3}$ has been considered to be the threshold of overtraining (Vervoorn et al.1991). Poor performance outcomes and suboptimal training adaptations have been reported in both soccer athletes (Kraemer et al.2004) and tactical athletes (Chicharro et al.1998) with a low T:C ratio. As other hormones moderate physiological adaptations to training, especially in female athletes, monitoring other hormones, such as SHBG or DHEA-S in relation to cortisol may provide additional insights into the anabolic to catabolic balance in both male and female athletes.

Dehydroepiandrosterone is a precursor hormone to both estrogen and testosterone. In addition to affecting body composition (Ha"kkinen et al.1988) in athletes, changes in DHEA in relation to cortisol have been reported to be a useful marker of susceptibility to overtraining in the female athlete (Bouget et al.2006 ; Filaire et al.1998). Similarly, Sex Hormone Binding Globulin (SHBG) is a useful indicator of training status and performance (strength and rate of force development) (Fahrner et al.1998). SHBG transports hormones such as testosterone in the body and increases in response to exercise training in both male and female athletes. Increased SHBG is believed to protect sex hormones from being degraded by protecting the biologically active free sex hormones in circulation. Increased SHBG and decreased testosterone may indicate insufficient recovery (Ha“kkinen et al.1988). Low SHBG may merely represent an individual's chronic diet (Adlercreutz 1990); diets high in fat and protein may be associated with low levels of SHBG and high levels of sex hormones (Adlercreutz 1990) and may be considered a sign of suboptimal capacity to adapt to training (Urhausen, et al.1995).

Other key hormones inform us about training adaptations. These include GH, IGF-1, and LH. Growth hormone $(\mathrm{GH})$ stimulates anabolism by promoting muscle protein synthesis and inhibiting protein breakdown. Growth hormone $(\mathrm{GH})$ concentrations have been correlated to exercise volume and intensity. Growth hormone increases levels of circulating IGF-I, both of which hormones are involved in muscle mass regulation, making IGF-1 and GH together potentially useful biomarkers. Luteinizing hormone ( $\mathrm{LH})$ is associated with reproductive function in men and women. Luteinizing hormone may be another useful marker to detect overtraining or insufficient energy intake.

\section{Protein-Amino Acids}

Athletes require greater daily intakes of protein (in the range of $1.3-1.8 \mathrm{~g} . \mathrm{kg}^{-1} \cdot \mathrm{d}^{-1}$ ) to maximize muscle protein synthesis as compared to the general population. The markers of nitrogen balance 


\section{Journal of Exercise Science \& Physiotherapy, Vol. 14, No. 1 (January to June), 2018 ISSN: 0973-2020 (Print) I I OR Impact Factor = 5.23 UGC Approved [no.7485] ISSN: 2454-6089 (Online)}

(e.g., urea nitrogen) are important for assessing the nutritional status of an athlete, but a number of specific amino acids can reveal information about protein synthesis, nutrition, and fatigue. For example, the branched-chain amino acids (BCAA), leucine, isoleucine, and valine, increase the rates of protein synthesis and degradation in resting human muscle (Blomstrand et al.2006). Branched-chain amino acids (BCAA) levels have been informative about whether BCAA supplementation is directly affecting skeletal muscle protein synthesis signaling (Apro et al.2010). With some special considerations for measurement (Tom and Nair 2006), BCAA can also indicate whether diet, stress, or disease states are affecting an athlete's skeletal muscle. There are a few other examples in which specific amino acids may indicate muscle status based on their unique roles in skeletal muscle. The amino acid taurine is not incorporated into protein, but is abundant in muscle tissue and is needed for the differentiation and growth of skeletal muscle. Taurine deficiency can impair muscle development, structure, and function (Spriet and Whitfield 2015). Researchers have interpreted elevated taurine levels, perhaps because of release from muscle fibers, as a marker of damage or impaired muscle function (Cuisinier et al.2001; Ward et al.1999). Others have used urine excretion of taurine as a biomarker in athletes (Corsetti et al. 2016).

Another amino acid, glycine, is involved in the biosynthesis of heme, creatine, nucleic acids, and uric acid (Wang et al.2013), deficiencies in which may affect various aspects of the metabolic pathways. Other amino acid patterns (e.g., elevated tryptophan, decreased glutamine) have been associated with fatigue and suboptimal training capacity in athletes (Smith and Norris 2000; Castell et al. 1999; Kingsbury et al. 1998) and suggest specific amino acids that may serve as biomarkers of muscle quality/ status. While some amino acids change in response to acute exercise (Refsum et al.1979), monitoring resting amino acids across a season as part of a comprehensive panel under similar conditions (i.e., fasted, in the morning, before training, the day after a rest day or similar training day) may provide insights into training (Einspahr and Tharp 1989) and fatigue (Kingsbury et al.1998).

\section{Recovery from Training (Urea Nitrogen and Creatine Kinase)}

After muscle-damaging exercise, the enzyme Creatine kinase (CK) leaks from the muscle into the circulation (Koch, et al. 2014; Mougios 2007). It is typical for athletes to have elevated Creatine kinase (CK) during training, with reference ranges of $82-1,083 \mathrm{U} . \mathrm{L}^{-1}$ in male and $47-513 \mathrm{U} . \mathrm{L}^{-1}$ in female athletes suggested as athletic norms (Mougios 2007). Monitoring CK levels during training in comparison with baseline levels may help athletes to monitor muscle status. Creatine kinase levels peak approximately 24 hours after damaging exercise such as heavy strength training, but may remain elevated up to 7 days after exercise. Chronically elevated CK may indicate insufficient recovery. Because other components of muscle such as myoglobin may leak into circulation during muscle damage (peak 1-3 hours after exercise), and urea nitrogen can indicate overall protein synthesis vs. breakdown (Hong and Lien 1984), using all 3 markers to determine an athlete's muscle status during training and recovery will be useful to athletes, coaches, and clinicians.

\section{References}

Adlercreutz, H. 1990.Western diet and Western diseases: Some hormonal and biochemical mechanisms and associations. Scand J Clin Lab Invest Suppl 201: 3-23.

Apro, Wand Blomstrand, E.2010.Influence of supplementation with branched-chain amino acids in combination with resistance exercise on p70S6 kinase phosphorylation in resting and exercising human skeletal muscle. Acta Physiol (Oxf) 200: 237-248.

Banfi, G, Marinelli, M, Roi, GS, and Agape, V.1993.Usefulness of free testosterone/cortisol ratio during a season of elite speed skating athletes. Int J Sports Med 14: 373-379.

Blomstrand, E, Eliasson, J, Karlsson, HK, and Ko“hnke, R.2006.Branched chain amino acids activate key enzymes in protein synthesis after physical exercise. J Nutr 136(1 Suppl): 269S-73S. 
Bouget, M, Rouveix, M, Michaux, O, Pequignot, JM, and Filaire, E.2006.Relationships among training stress, mood and dehydroepiandrosterone sulphate/cortisol ratio in female cyclists. J Sports Sci 24: 1297-1302.

Castell, LM, Yamamoto, T, Phoenix, J, and Newsholme, EA.1999.The role of tryptophan in fatigue in different conditions of stress. Adv Exp Med Biol 467: 697-704.

Chicharro, JL, Lo' pez-Mojares, LM, Luc1'a, A, Pe' rez, M, Alvarez, J, Labanda, P, Calvo, F, and Vaquero, AF.1998.Overtraining parameters in special military units. Aviat Space Environ Med 69: 562-568.

Corsetti, R, Barassi, A, Perego, S, Sansoni, V, Rossi, A, Damele, CA, Melzi D’Eril, G, Banfi, G, and Lombardi, G.2016.Changes in urinary amino acids excretion in relationship with muscle activity markers over a professional cycling stage race: In search of fatigue markers. Amino Acids 48: 183-192.

Cuisinier, C, Ward, RJ, Francaux, M, Sturbois, X, and de Witte, P.2001.Changes in plasma and urinary taurine and amino acids in runners immediately and $24 \mathrm{~h}$ after a marathon. Amino Acids 20: 13-23.

Einspahr, KJ and Tharp, G.1989.Influence of endurance training on plasma amino acid concentrations in humans at rest and after intense exercise. Int J Sports Med 10: 233-236.

Fahrner, CL and Hackney, AC.1998.Effects of endurance exercise on free testosterone concentration and the binding affinity of sex hormone binding globulin (SHBG). Int J Sports Med 19: $12-15$.

Filaire, E, Duche, P, and Lac, G.1998.Effects of amount of training on the saliva concentrations of cortisol, dehydroepiandrosterone and on the dehydroepiandrosterone: Cortisol concentration ratio in women over 16 weeks of training. Eur J Appl Physiol Occup Physiol 78: 466-471.

Ha"kkinen, K, Pakarinen, A, Ale' n, M, Kauhanen, H, and Komi, PV.1988.Neuromuscular and hormonal adaptations in athletes to strength training in two years. J Appl Physiol (1985) 65: 2406-2412.

Hong, CZ and Lien, IN.1984.Metabolic effects of exhaustive training of athletes. Arch Phys Med Rehabil 65: 362-365.

Kingsbury, KJ, Kay, L, and Hjelm, M.1998.Contrasting plasma free amino acid patterns in elite athletes: Association with fatigue and infection. Br J Sports Med 32: 25-32.

Koch, AJ, Pereira, R, and Machado, M.2014.The creatine kinase response to resistance exercise. J Musculoskelet Neuronal Interact 14: 68-77.

Kraemer, WJ, French, DN, Paxton, NJ, Ha“kkinen, K, Volek, JS, Sebastianelli, WJ, Putukian, M, Newton, RU, Rubin, MR, Go' mez, AL, Vescovi, JD, Ratamess, NA, Fleck, SJ, Lynch, JM, and Knuttgen, HG.2004.Changes in exercise performance and hormonal concentrations over a big ten soccer season in starters and nonstarters. J Strength Cond Res 18: $121-128$.

Mougios, V.2007. Reference intervals for serum creatine kinase in athletes. Br J Sports Med 41: 674-678.

Refsum, HE, Gjessing, LR, and Stromme, SB.1979. Changes in plasma amino acid distribution and urine amino acids excretion during prolonged heavy exercise. Scand J Clin Lab Invest 39: 407-413.

Smith, DJ and Norris, SR.2000.Changes in glutamine and glutamate concentrations for tracking training tolerance. Med Sci Sports Exerc 32: 684-689.

Spriet, LL and Whitfield, J.2015.Taurine and skeletal muscle function. Curr Opin Clin Nutr Metab Care 18: 96-101.

Tom, A and Nair, KS.2006. Assessment of branched-chain amino Acid status and potential for biomarkers. J Nutr 136(1 Suppl):324S-330S.

Urhausen, A, Gabriel, H, and Kindermann, W.1995. Blood hormones as markers of training stress and overtraining. SportsMed 20: 251-276. 